\title{
COMPLEMENTING MAPS, CONTINUATION AND GLOBAL BIFURCATION
}

\author{
BY P. M. FITZPATRICK, I. MASSABO AND J. PEJSACHOWICZ
}

\begin{abstract}
We state, and indicate some of the consequences of, a theorem whose sole assumption is the nonvanishing of the LeraySchauder degree of a compact vector field, and whose conclusions yield multidimensional existence, continuation and bifurcation results.
\end{abstract}

Complementing maps and the Theorem. Let $X$ be a Banach space, $m$ be a positive integer, and $O \subseteq \mathbf{R}^{m} \times X$ be open. Suppose $f: O \rightarrow X$ is an $m$-parameter compact vector field: i.e. $f(\lambda, x)=x-F(\lambda, x)$, for $(\lambda, x) \in O$, where $F$ is continuous and maps bounded sets into relatively compact sets. A continuous map $g: O \rightarrow \mathbf{R}^{m}$, which maps bounded sets into bounded sets, will be called a complement for $f: O \rightarrow X$ provided that the Leray-Schauder degree, $\operatorname{deg}((g, f), O, 0)$, is defined and nonzero: $(g, f)((\lambda, x)) \equiv(g(\lambda, x), f(\lambda, x))$, for $(\lambda, x) \in O$, and since $O$ is not assumed to be bounded, "defined" means $(g, f)^{-1}(0)$ is compact.

By cohomology we will mean Cech cohomology with integral coefficients. By dimension of a topological space we mean the Cech-Lebesgue covering dimension, and if $p \in A$, the space $A$ will be said to have dimension at least $m$ at $p$ provided that each neighborhood, in $A$, of $p$ has dimension at least $m$.

THEOREM. Let $X$ be a Banach space, $m$ be a positive integer, and $O \subseteq$ $\mathbf{R}^{m} \times X$ be open. Suppose that $f: O \rightarrow X$ is complemented by $g: O \rightarrow \mathbf{R}^{m}$. Then there exists a closed connected subset, $C$, of $f^{-1}(0)$, whose dimension at each point is at least $m$, and (*) whenever $K$ is a compact subset of $C$, with $g^{-1}(0) \cap C \subseteq K$, the map of pairs $g:(C, C-K) \rightarrow\left(\mathbf{R}^{m}, \mathbf{R}^{m}-0\right)$ induces a nontrivial map in the mth cohomology group. In particular, $C \cap g^{-1}(0) \neq \varnothing$ and either $C$ is unbounded or $\bar{C} \cap \partial O \neq \varnothing$. In the case when $f$ and $g$ are defined on $\bar{O}$ with $f^{-1}(0) \cap g^{-1}(0) \cap \partial O=\varnothing, C$ also has the following properties: if $C$ is bounded, then $\operatorname{dim}(\bar{C} \cap \partial O) \geq m-1$, when $m>1$, and $\bar{C} \cap \partial O$ has at least two points, when $m=1$; if $g: f^{-1}(0) \cap \bar{O} \rightarrow \mathbf{R}^{m}$ is proper and $\operatorname{dim}(\bar{C} \cap \partial O)<m-1$, then $g(\bar{C})=\mathbf{R}^{m}$.

Skeleton of the Proof. Since $\operatorname{deg}((g, f), O, 0) \neq 0$, by using the cupproduct in cohomology, it follows that whenever $K$ is compact and $g^{-1}(0) \subseteq$ $K \subseteq f^{-1}(0)$ the map $g:\left(f^{-1}(0), f^{-1}-K\right) \rightarrow\left(\mathbf{R}^{m}, \mathbf{R}^{m}-0\right)$ is cohomologically nontrivial. Passing to the limit over all such $K$ 's we obtain a nontrivial class, $\xi$, in the $m$ th Cech cohomology group with compact supports of $f^{-1}(0)$. The continuity of Cech theory enables us to choose a set, $C$, which is minimal

Received by the editors May 18, 1982.

1980 Mathematics Subject Classification. Primary 47H15, 35B32, 45 G05.

(c) 1983 American Mathematical Society $0273-0979 / 83 \$ 1.00+\$ .25$ per page 
among the closed subsets of $f^{-1}(0)$ to which $\xi$ restricts nontrivially. This $C$ has the properties claimed.

Some consequences of the Theorem. In what follows, $f: O \subseteq \mathbf{R}^{m} \times X \rightarrow X$ is an $m$-parameter compact vector field.

1. Continuation under global hypotheses. Let $\lambda_{0} \in \mathbf{R}^{m}$ and let $f_{\lambda_{0}}$ be the section of $f$ over the slice $O_{\lambda_{0}}$. One shows that if $\operatorname{deg}\left(f_{\lambda_{0}}, O_{\lambda_{0}}, 0\right) \neq 0$ then $f: O \rightarrow X$ is complemented by $g: O \rightarrow \mathbf{R}^{m}$ defined by $g(\lambda, x)=\lambda-\lambda_{0}$. Thus the theorem furnishes a description of how $f^{-1}(0)$ emanates from $O_{\lambda_{0}}$. This is a multidimensional refinement of the Leray-Schauder continuation principle (see $[4,6$ and 7$]$ ).

2. Continuation under local hypotheses. Let $\left(\lambda_{0}, x_{0}\right) \in O$ and suppose that the map $x \rightarrow f\left(\lambda_{0}, x\right)$ has a Fréchet derivative, $L$, at $x=x_{0}$. Assume $L \in$ $\mathcal{L}(X, X)$ is invertible. Then, letting $U=O-\left\{\left(\lambda_{0}, x\right) \mid x \neq x_{0}, f\left(\lambda_{0}, x\right)=0\right\}$, one shows that $f: U \rightarrow X$ is complemented by $g: U \rightarrow \mathbf{R}^{m}$ defined by $g(\lambda, x)=\lambda-\lambda_{0}$. Thus, there is an $m$-dimensional connected subset, $C$, of $f^{-1}(0) \cap U$, which contains $\left(\lambda_{0}, x_{0}\right)$, and which is either unbounded or $\bar{C} \cap\left\{\partial O \cup\left\{\left(\lambda_{0}, x\right) \mid x \neq x_{0}, f\left(\lambda_{0}, x\right)=0\right\}\right\} \neq \varnothing$. Another global version of the implicit function theorem was obtained in [3].

3. Nonlinear perturbation of linear Fredholm operators. Let $\Omega \subseteq \mathbf{R}^{2}$ be simply connected, open and bounded, with $\partial \Omega$ a smooth closed curve. Suppose $\tau: \partial \Omega \rightarrow S^{1}$ is smooth and such that the winding number of $\tau: \partial \Omega \rightarrow S^{1}$ equals $-k<0$. Given $\phi, \psi: \bar{\Omega} \times \mathbf{R}^{2} \rightarrow \mathbf{R}$ we consider the following nonlinear Riemann-Hilbert problem: find $u, v: \bar{\Omega} \rightarrow \mathbf{R}$ such that, if $\tau=\left(\tau_{1}, \tau_{2}\right)$

$$
\text { (i) }\left\{\begin{array}{l}
u_{x}-v_{y}=\phi(x, y, u, v), \\
v_{x}+u_{y}=\psi(x, y, u, v)
\end{array} \text { in } \Omega,\right.
$$

$$
\text { (ii) } u \tau_{1}-v \tau_{2}=0 \text { on } \partial \Omega \text {. }
$$

Let $\alpha \in(0,1)$ be such that $\psi$ and $\phi$ lie in $C^{1+\alpha}(\bar{\Omega} \times A, \mathbf{R})$ for each bounded subset $A$ of $\mathbf{R}^{2}\left(C^{1+\alpha}\right.$ denotes the usual Schauder space). Under the assumption that $\psi(x, y, 0,0)=\phi(x, y, 0,0)=0$, for each $(x, y) \in \bar{\Omega}$, it follows that for each $r>0,\left\{(u, v) \in C^{1+\alpha}\left(\bar{\Omega}, \mathbf{R}^{2}\right) \mid(u, v)\right.$ solves $\left.\mathrm{R}-\mathrm{H},\|(u, v)\|_{1+\alpha}=r\right\}$ has dimension at least $2 k$.

Let $W=\left\{(u, v) \in C^{1+\alpha}\left(\bar{\Omega}, \mathbf{R}^{2}\right) \mid(u, v)\right.$ satisfies (ii) $\}$, and let $L: W \rightarrow$ $C^{\alpha}\left(\bar{\Omega}, \mathbf{R}^{2}\right)$ be the linear operator defined by the left-hand side of (i). Choose $z_{1}, \ldots, z_{k}$ in $\Omega$ and define $g: W \rightarrow \mathbf{R}^{2 k+1}$ by

$$
g((u, v))=\left(u\left(z_{1}\right), v\left(z_{1}\right), \ldots, u\left(z_{k}\right), v\left(z_{k}\right), \int_{\partial \Omega}\left[\tau_{1} v+\tau_{2} u\right] d s\right) .
$$

Letting $X=g^{-1}(0)$, the linear theory (see [10]) implies $L: X \rightarrow C^{\alpha}\left(\bar{\Omega}, \mathbf{R}^{2}\right)$ has an inverse, $T$, and $W=V \oplus X$, with $\operatorname{dim}(V)=2 k+1$.

If we rewrite $(\mathrm{R}-\mathrm{H})$ as $f((u, v)) \equiv T(L-H)((u, v))=0$, one shows that $f: V \oplus X \rightarrow X$ is complemented by $g$ on each ball about the origin in $W$, and so we can apply the Theorem.

4. Global bifurcation. For simplicity, we assume $O=\mathbf{R}^{m} \times X$. We assume $\mathbf{R}^{m} \times\{0\} \subseteq f^{-1}(0)$, and call $\mathbf{R}^{m} \times\{0\}$ the trivial solutions of $f$. Suppose $\alpha, \beta \in$ $\mathbf{R}^{m}$ are such that $(\alpha, 0)$ and $(\beta, 0)$ are not bifurcation points of $f^{-1}(0)$ and that 
$\operatorname{ind}\left(f_{\alpha}, 0\right) \neq \operatorname{ind}\left(f_{\beta}, 0\right)$, where "ind" denotes the Leray-Schauder index. Then, if $\Gamma$ is any open curve (i.e. homeomorphic image of $\mathbf{R}$ ) in $\mathbf{R}^{m} \times\{0\}$ which passes through $(\alpha, 0)$ and $(\beta, 0)$, there exists a connected set, $C$, of nontrivial zeros of $f$, whose dimension at each point is at least $m$, which intersects the segment, $\overline{(\alpha, 0),(\beta, 0)}$, of $\Gamma$, determined by $(\alpha, 0)$ and $(\beta, 0)$, and either $C$ is unbounded or $\bar{C}$ intersects $\Gamma-\{\overline{(\alpha, 0),(\beta, 0)}\}$.

When $\alpha=0, \beta=(1,0, \ldots)$ and $\Gamma$ is the line through $\alpha$ and $\beta$ the proof runs as follows. Choose $r>0$ such that $f(\lambda, x) \neq 0$ when $0<\|x\| \leq r$ and either $|\lambda| \leq 3 r$ or $|\lambda-\beta| \leq 3 r$. Let $h: \mathbf{R} \rightarrow[0, r]$ be continuous, vanish outside of $[-r, 1+r]$, and equal $r$ on $[r, 1-r]$. Then define $g: \mathbf{R}^{m} \times X \rightarrow \mathbf{R}^{m}$ by $g\left(\lambda_{1}, \ldots, \lambda_{m}\right)=\left(\|x\|^{2}-\left(h\left(\lambda_{1}\right)\right)^{2}, \lambda_{2}, \ldots, \lambda_{m}\right)$.

One shows that if $U=\mathbf{R}^{m} \times\{X-\{0\}\}$, then $\operatorname{deg}((g, f), U, 0)=\operatorname{ind}\left(f_{\beta}, 0\right)-$ $\operatorname{ind}\left(f_{\alpha}, 0\right)$, and so $g$ complements $f$ on $U$. So we extract the subset, $C$, of $f^{-1}(0) \cap U$, having the properties in the conclusion of the Theorem. Conclusion (*) implies our assertions regarding $\bar{C} \cap \Gamma$.

This bifurcation result yields the principle abstract global bifurcation results of [9 and 1]. J. Ize (see [8]) has given a proof of the bifurcation theorem in [9] using a map similar to the above $g$.

REMARK. In the definition of complementing map if one replaces the Leray-Schauder degree by the Browder-Petryshyn degree for $A$-proper mappings (see [5]) the Theorem still holds. We believe that approximation results similar to those used in [2] will also yield the Theorem when $F$ is assumed to be condensing.

\section{REFERENCES}

1. J. C. Alexander and S. S. Antman, Global and local behaviour of bifurcating multidimensional continua of solutions of multiparameter eigenvalue problems, Arch. Rational Mech. Anal. 76 (1981), 339-354.

2. J. C. Alexander and P. M. Fitzpatrick, The homotopy of certain spaces of non-linear operators and its relation to global bifurcation of the fixed-points of parametrized condensing maps, J. Funct. Anal. 34 (1979), 87-106.

3. J. C. Alexander and J. A. Yorke, The implicit function theorem and the global methods of cohomology, J. Funct. Anal. 21 (1976), 330-339.

4. F. E. Browder, On continuity of fixed-points under deformations of continuous mappings, Summa Brazilia Math. 4 (1960), 183-190.

5. F. E. Browder and W. V. Petryshyn, Approximation methods and the generalized topological degree for non-linear mappings in a Banach space, J. Funct. Anal. 3 (1969), 217-245.

6. J. Leray and J. Schauder, Topologie et équations fonctionnelles, Ann. Sci. École Norm. Sup. 51 (1934), 45-78.

7. I. Massabo and J. Pejsachowicz, On the connectivity properties of the solution set of parametrized families of compact vector fields, J. Funct. Anal. (to appear).

8. L. Nirenberg, Topics in nonlinear functional analysis, Courant Inst. Lecture Notes, 1975.

9. P. H. Rabinowitz, Some global results for nonlinear eigenvalue problems, J. Funct. Anal. 7 (1971), 487-513.

10. W. L. Wendland, Elliptic systems in the plane, Monographs and Studies in Mathematics, no. 3, Pitman, New York, 1979.

Department OF MATHEMATICS, UNIVERSity OF MARYland, COLlEge PARK, MARYLAND 20742

Dipartimento di MATEMATICA, UNIVERSit ḋ della CALABRIA, ARCAVACATA DI RENDE, CALABRIA, ITALY 
